\title{
A Practical Solution Model for Transient Pressure Behavior of Multistage Fractured Horizontal Wells with Finite Conductivity in Tight Oil Reservoirs
}

\author{
Pin Jia $\mathbb{D}^{1,2}$ Defeng Wu, ${ }^{3}$ Hengfei Yin, ${ }^{4}$ Zhuang Li, ${ }^{5}$ Linsong Cheng $\mathbb{D}^{1,2}$ and Xianzhe $\mathrm{Ke}^{1,2}$ \\ ${ }^{1}$ State Key Laboratory of Petroleum Resources and Prospecting, Beijing 102249, China \\ ${ }^{2}$ China University of Petroleum (Beijing), Beijing 102249, China \\ ${ }^{3}$ CNPC Chuanqing Drilling Engineering Co. Ltd., Chengdu 610051, China \\ ${ }^{4}$ PetroChina Research Institute of Petroleum Exploration and Development, Beijing 100083, China \\ ${ }^{5}$ CNOOC China Limited, Shenzhen Branch, Shenzheng 518067, China
}

Correspondence should be addressed to Pin Jia; jiapin1990@163.com

Received 15 March 2021; Revised 17 May 2021; Accepted 11 June 2021; Published 21 June 2021

Academic Editor: Mohammad Sarmadivaleh

Copyright (c) 2021 Pin Jia et al. This is an open access article distributed under the Creative Commons Attribution License, which permits unrestricted use, distribution, and reproduction in any medium, provided the original work is properly cited.

\begin{abstract}
Fractured horizontal wells have been widely used to develop unconventional oil and gas reservoirs. In previous studies, most studies on the transient pressure behavior of multistage horizontal wells were based on the assumption of single porosity medium, in which the coupling relationship of natural fractures and artificial fractures was not taken into account or artificial fractures were assumed to be infinitely conductive. In this paper, the fracture is finite conductive, which means that there is flow resistance in the fracture. Based on point-source method and superposition principle, a transient model for multistage fractured horizontal wells, which considers the couple of fracture flow and reservoir seepage, is built and solved with the Laplace transformation. The transient pressure behavior in multistage fractured horizontal wells is discussed, and effects of influence factors are analyzed. The result of this article can be used to identify the response characteristic of fracture conductivity to pressure and pressure differential and provide theoretical basis for effective development of tight oil reservoirs. The findings of this study can help for better understanding of transient pressure behavior of multistage fractured horizontal wells with finite conductivity in tight oil reservoirs.
\end{abstract}

\section{Introduction}

Tight oil reserves are always be seen as the alternative resources for conventional oil reservoirs due to their abundant reserves. However, the physical property of tight oil reservoirs is extremely poor, and therefore, almost no commercial oil and gas flow can be obtained under conventional recovery method. Horizontal well and hydraulic fracturing are proved to be the key techniques for efficiently developing unconventional oil and gas reservoirs $[1,2]$. As the artificial fractures caused by massive hydraulic fracturing and natural fractures form a complicated fracture system near wellbore, the percolation mechanism and production performance of multistage fractured horizontal wells are different from conventional fractured horizontal wells. It is extremely necessary to study the percolation mechanism and production performance of multistage fractured horizontal wells in tight oil reservoirs, in order to maximize its productivity.

A variety of researches have been made on behavioral characteristics and evaluation and productivity of fractured horizontal wells since the 1990s. Early studies were mainly based on single fracture caused by conventional fracturing and analyzed development effect, transient pressure, and behavioral characteristics of fractured horizontal wells [3-8]. Fractured horizontal wells have recently been widely used to develop unconventional reservoirs and lead to a more complicated percolation theory. Wang et al. [9] built the coupling model for stepwise inflow into fractures, stepwise inflow into horizontal wellbore, and percolation in the reservoir for fractured horizontal wells. Lian et al. [10] presented a transient model in which fractured horizontal wellbore is 


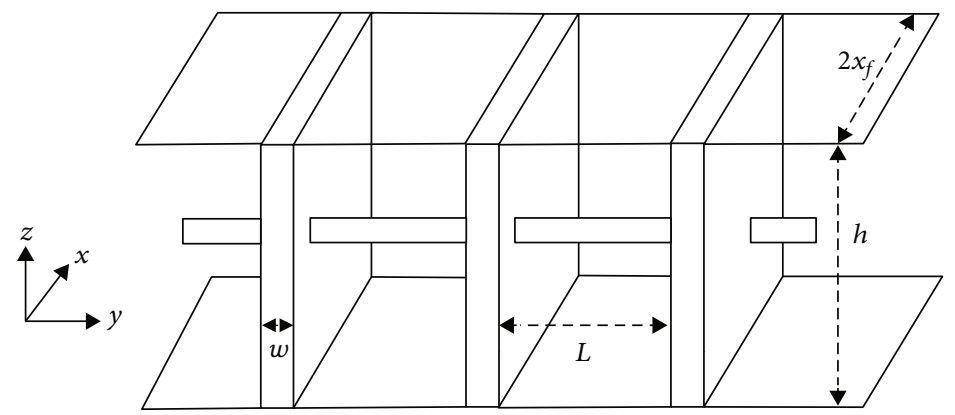

FIGURE 1: The physical model for multistage fractured horizontal wells.

coupled with a box anisotropic reservoir of low permeability. By using Green function and Newman integral principle, Sun et al. [11] built a transient model for fractured horizontal wells in which the reservoir is coupled with horizontal wellbore, and the flow is fed by the matrix and the artificial fractures. Valkó and Amini [12] and Zhu et al. [13] presented a productivity evaluation model for fractured horizontal wells in a box reservoir of low permeability with discrete volume-source method and presented a model for complicated fractures near multistage fractured horizontal wellbores in single porosity medium with slab-source method, assuming that the dominant flow regime in the fractures is radial flow [14]. Yao et al. presented a semianalytical model for multistage horizontal wells in which the pressure drawdown in horizontal wellbore is coupled with the reservoir, taking account of the finite conductivity of the fractures with point-source function method [15]. Researches above are all based on conventional plane fracture, and fail to taking account of the coupling of natural fractures and artificial fractures. Zhao et al. [16] and Guo et al. [17] presented a transient percolation model for multistage horizontal wells in shale gas reservoirs with dualporosity medium assuming that the artificial fractures are infinitely conductive. Brown et al. [18] and Imad et al. [19] developed a tri-linear model for multi-stage fractured horizontal wells with composite zoning, assuming the near wellbore region to be dual-porosity medium. Based on the trilinear model. Stalgorova and Mattar [20, 21] proposed composited models with three zones and five zones, respectively. The abovementioned method to deal with natural fractures is inconsistent with the actual microseismic monitoring materials, and the flow in the fractures is not taken into account $[22,23]$.

On basis of the current research of fractured horizontal wells, this article builds a transient model in dual-porosity medium for multistage horizontal wells in tight oil reservoirs which takes into account of finite conductivity in the artificial fractures. The model is solved by using the source function method and superposition principle. Meanwhile, type curves are plotted to identify different flow regimes, and influential factors of transient pressure are analyzed as well. The structure of the article is as follows. First, the physical model is presented. Then, a mathematical model is built on the basis of the physical model, and the related characteristics of the curve are further analyzed. Finally, it is applied in the actual block.

\section{Physical Model}

After tight oil reservoirs are artificially fractured, the natural fractures and artificial fractures form a complicated fracture system, and the whole reservoir can be viewed as a dualporosity medium composed of the matrix and the fracture system. The transient flow from the reservoir to the wellbore can be divided into two parts: the flow from the matrix to the fractures and the flow in the fractures. The pressure at any point in the formation at any time and the flow rate of every fracture grid can be obtained by coupling the pressure and the flow rate of the two flow regimes at the fracture surface. The physical model is shown in Figure 1. Some basic assumptions are given as follows:

(1) The reservoir is homogeneous and laterally infinite, with uniform thickness and impermeable parallel boundaries at the top and the bottom. The thickness of the reservoir is $h$, the porosity is $\phi$, the average permeability is $k$, and the artificial permeability is $k_{f}$

(2) The reservoir rock and fluid are slightly compressible. The total compressibility is $c_{t}$, and the viscosity of the fluid is $\mu$. The storage of the fractures is neglected

(3) The artificial fractures cut across the entire reservoir and traverse along the direction of the maximum horizontal principal stress. There are $M$ artificial fractures which are uniformly spaced. The distance between each two neighboring fractures is $L$. Half-length of these fractures is, respectively, $x_{f 1}, x_{f 2}, \cdots, x_{f \mathrm{M}}$, and the conductivity is $k_{f} \cdot w$

\section{Mathematical Model}

3.1. Reservoir Flow Model. The top and bottom boundaries of the reservoir are impermeable, and the reservoir is laterally infinite. The natural microfractures and the artificial fractures form a fracture network. Half-length of the artificial fractures is discretized into $N$ segments equally as shown in Figure 2. The coordinate of the midpoint of discrete segment $(i, j)$ is $\left(x_{m i j}, y_{m i j}\right)$, and the coordinate of the starting point and the end point is $\left(x_{i \mathrm{j}}, y_{i, j}\right)$ and $\left(x_{i j+1}, y_{i, j}\right)$, respectively. Therefore, the pressure at the number $i$ discrete fracture segment caused by any discrete fracture segment in any artificial fracture in the reservoir is 


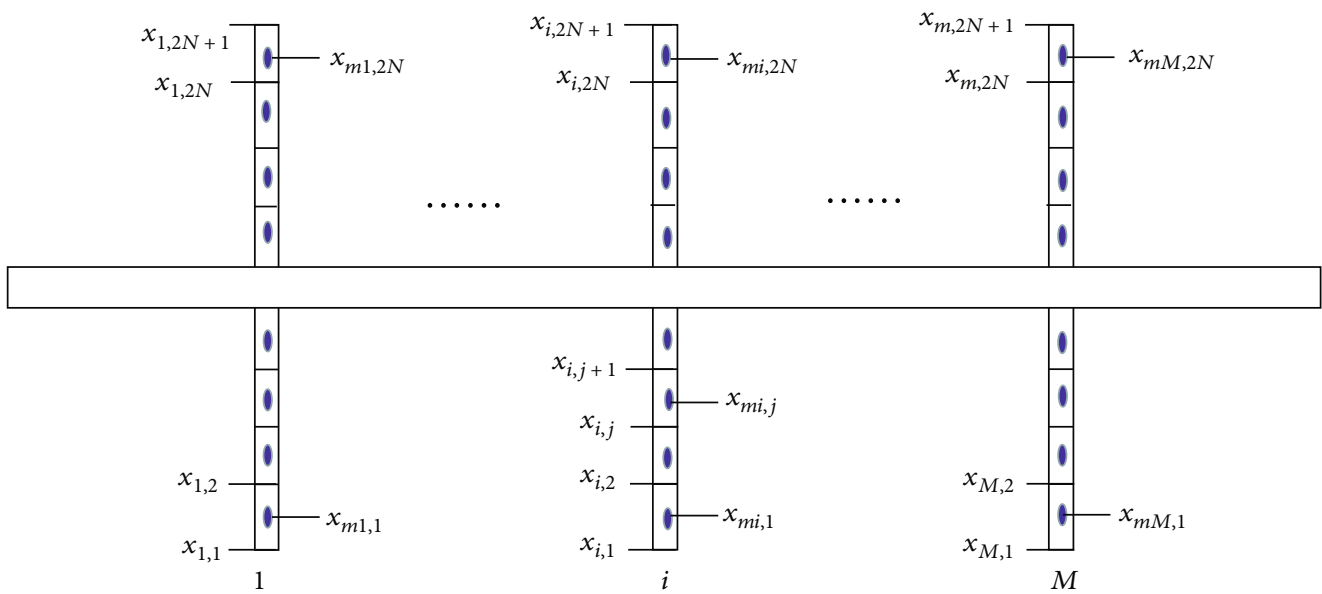

Figure 2: Discretion of fracture network.

$$
p_{D i}\left(t_{D}\right)=\sum_{j=1}^{M \cdot 2 N} \int_{0}^{t_{D}} q_{f D j}(\tau) p_{D i, j}^{\prime}\left(x_{D}, y_{D} ; t_{D}-\tau\right) d \tau .
$$

The total flow rate from the reservoir to each discrete segment in each fracture is the production rate of the horizontal well:

$$
\sum_{j=1}^{M \cdot 2 N} q_{f D j}\left(t_{D}\right)=1
$$

Transform Eq. (1) and Eq. (2) to Laplace space as follows:

$$
\begin{gathered}
\sum_{j=1}^{M \cdot 2 N} \bar{q}_{f D i, j}(s)=\frac{1}{s}, \\
\bar{p}_{D i}(\mathrm{~s})=\sum_{j=1}^{M \cdot 2 N} s \bar{q}_{D i} \bar{p}_{D i, j}\left(x_{D}, y_{D}\right) .
\end{gathered}
$$

According to the study of Ozkan and Raghavan [22], pressure drawdown at a particular point in the reservoir caused by linear source in Laplace domain space is

$$
\begin{aligned}
\bar{p}_{D i, j}\left(x_{D}, y_{D}\right)= & \frac{1}{s \Delta x_{f D}} \int_{x_{D i, j}}^{x_{D i, j+1}} K_{0}(\sqrt{f(u)} \\
& \left.\cdot \sqrt{\left(x_{D}-x_{m D j}-x\right)^{2}+\left(y_{D}-y_{m D j}\right)^{2}}\right) d x,
\end{aligned}
$$

where the dimensionless variables are defined as follows:

$$
\begin{gathered}
x_{D}=\frac{x}{L}, \\
y_{D}=\frac{y}{L}, \\
x_{w D}=\frac{x_{w}}{L}, \\
y_{w D}=\frac{y_{w}}{L} .
\end{gathered}
$$

There are $M \cdot 2 N$ equations satisfying Eq. (4). The matrix form is

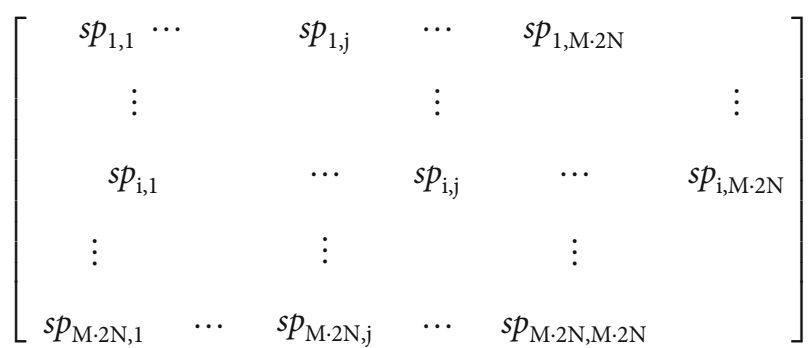

$$
\left[\begin{array}{c}
q_{f D 1,1} \\
\vdots \\
q_{f D i, j} \\
\vdots \\
q_{f D M \cdot 2 N}
\end{array}\right]=\left[\begin{array}{c}
p_{f D 1,1} \\
\vdots \\
p_{f D i, j} \\
\vdots \\
p_{f D M \cdot 2 N}
\end{array}\right]
$$

3.2. Model for the Flow in the Fractures with Finite Conductivity. As dominant flow regime in the fracture is transient flow as shown in Figure 3, the one dimensional flow equation for one particular fracture can be expressed in a dimensionless form as Eq. (8): 


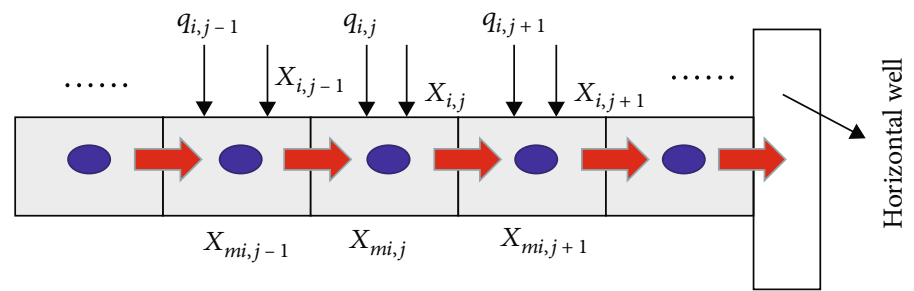

FIGURE 3: Schematic of discrete fracture segments.

$$
\frac{\partial^{2} p_{f D}}{\partial x_{D}^{2}}-\frac{2 \pi}{C_{f D}} \frac{q_{f D}\left(x_{D}, t_{D}\right)}{\Delta x_{f D}}=\frac{1}{\eta_{f D}} \frac{\partial p_{f D}}{\partial t_{D}}
$$

where $q_{f D}\left(x_{D}, t_{D}\right)$ is the dimensionless flow rate from the reservoir into one unit fracture length. The end of the fracture is impermeable, and the production of the producing well is constant. The total amount of the production from all the fractures into the wellbore is the production of the producing well. Transforming Eq. (8) in Laplace domain space, we can obtain the following equation:

$$
\frac{\partial^{2} \bar{p}_{f D}}{\partial x_{D}^{2}}-\frac{2 \pi}{C_{f D}} \frac{\bar{q}_{f D}\left(x_{D}, s\right)}{\Delta x_{f D}}=\frac{s}{\eta_{f D}} \bar{p}_{f D}
$$

Discretize Eq. (9) for the discrete segment as showed in Eq. (10) and rearrange it as follows:

$$
\begin{aligned}
& \frac{\bar{p}_{f D i, j-1}(s)-2 \bar{p}_{f D i, j}(s)+\bar{p}_{f D i, j+1}(s)}{\Delta x_{f D}{ }^{2}}-\frac{2 \pi}{C_{f D}} \frac{\bar{q}_{f D i, j}\left(x_{D i, j}, s\right)}{\Delta x_{f D}} \\
& =\frac{s}{\eta_{f D}} \bar{p}_{f D i, j} .
\end{aligned}
$$

Rearrange Eq. (10) as follows:

$$
\begin{gathered}
\bar{p}_{f D i, j-1}(s)-\left(2+\frac{s \cdot \Delta x_{f D}{ }^{2}}{\eta_{f D}}\right) \bar{p}_{f D i, j}(s)+\bar{p}_{f D i, j+1}(s) \\
=\frac{2 \pi \cdot \Delta x_{f D}}{C_{f D}} \bar{q}_{f D i, j}\left(x_{D i, j}, s\right) .
\end{gathered}
$$

The discrete fracture grids are divided into three types: the grid at the end of the fracture, interior grids, and the grids connecting with the wellbore. The discretized equation for the grids at the end of the fracture is

$\bar{p}_{f D i, 2}(s)-\left(1+\frac{s \cdot \Delta x_{f D}^{2}}{\eta_{f D}}\right) \bar{p}_{f D i, 1}(s)=\frac{2 \pi \cdot \Delta x_{f D}}{C_{f D}} \bar{q}_{f D i, 1}\left(x_{D i, 1}, s\right)$.
The equation for the interior fracture grid is

$$
\begin{gathered}
\bar{p}_{f D i, j-1}(s)-\left(2+\frac{s \cdot \Delta x_{f D}^{2}}{\eta_{f D}}\right) \bar{p}_{f D i, j}(s)+\bar{p}_{f D i, j+1}(s) \\
=\frac{2 \pi \cdot \Delta x_{f D}}{C_{f D}} \bar{q}_{f D i, j}\left(x_{D i j}, s\right) .
\end{gathered}
$$

The equation for the grids connecting with the wellbore is

$$
\begin{aligned}
& \frac{8}{3} \bar{p}_{w D}(s)-\left(4+\frac{s \cdot \Delta x_{f D}^{2}}{\eta_{f D}}\right) \bar{p}_{f D i, N}(s)+\frac{4}{3} \bar{p}_{f D i, N-1}(s) \\
& =\frac{2 \pi \cdot \Delta x_{f D}}{C_{f D}} \bar{q}_{f D i, N}\left(x_{D i, N}, s\right) .
\end{aligned}
$$

Tackling the boundary condition and combining the above $N$ equations result the following matrix equation for the flow in the fracture:

$$
\begin{aligned}
& {\left[\begin{array}{cccccccc}
-(1+\alpha) & 1 & 0 & \cdot & \cdot & \cdot & 0 & 0 \\
1 & -(2+\alpha) & 1 & \cdot & \cdot & \cdot & 0 & 0 \\
0 & 1 & -(2+\alpha) & . & . & . & 0 & 0
\end{array}\right.} \\
& \ddots \\
& \left.\begin{array}{lllll}
0 & 0 & 0 & \frac{4}{3} & -(4+\alpha)
\end{array}\right] \\
& \cdot\left[\begin{array}{c}
p_{f D i, 1} \\
p_{f D i, 2} \\
p_{f D i, 3} \\
\cdot \\
p_{f D i, N}
\end{array}\right]=\beta\left[\begin{array}{c}
q_{f D i, 1} \\
q_{f D i, 2} \\
q_{f D i, 3} \\
\cdot \\
\\
q_{f D i, N}
\end{array}\right]+\left[\begin{array}{c}
0 \\
0 \\
0 \\
\cdot \\
0 \\
-\frac{8}{3} p_{w D}
\end{array}\right] \text {, }
\end{aligned}
$$

where $\alpha=s \cdot \Delta x_{f D}{ }^{2} / \eta_{f D}$ and $\beta=2 \pi \cdot \Delta x_{f D} / C_{f D}$. The dimensionless variables are 


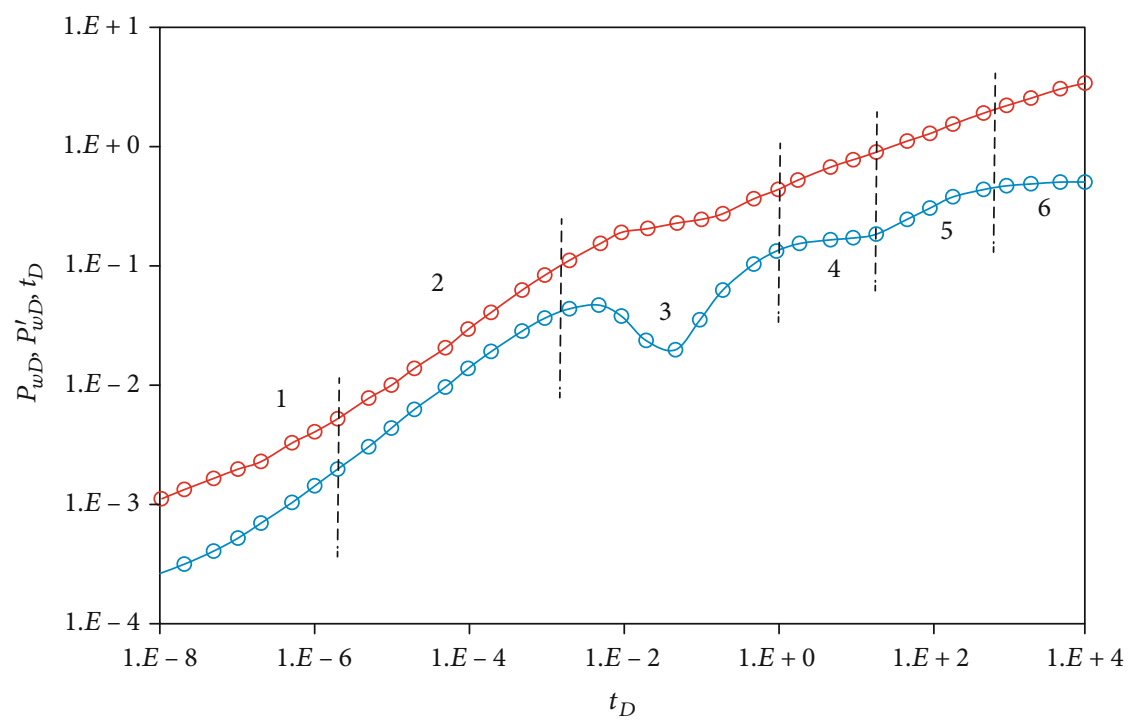

FIgURE 4: Type curves for multistage fractured horizontal wells in tight oil reservoirs.

$$
\begin{aligned}
t_{D} & =\frac{k t}{\phi \mu c_{t} L^{2}}, \\
\Delta x_{f D} & =\frac{\Delta x_{f}}{L}, \\
\eta_{f D} & =\frac{k_{f} \phi c_{t}}{k \phi c_{\mathrm{ft}}}, \\
C_{f D} & =\frac{k_{f} w}{k L}, \\
q_{f D} & =\frac{q_{f}}{q_{w}}, \\
p_{D} & =\frac{2 \pi k\left(p_{i}-p\right)}{\mu q_{w}} .
\end{aligned}
$$

3.3. Solution for the Coupling Model of the Reservoir and the Fractures. Combining the flow equations for the reservoir (Eq. (7)), the equation for the flow in the fracture (Eq. (15)) results in the following matrix equation for coupling flow:

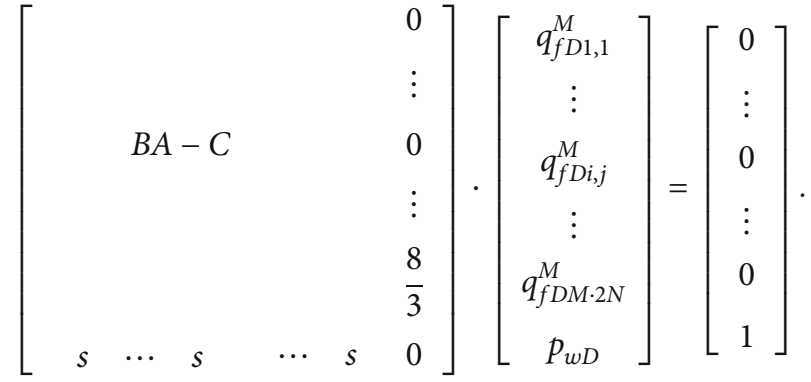

In which, the matrix $A$ is

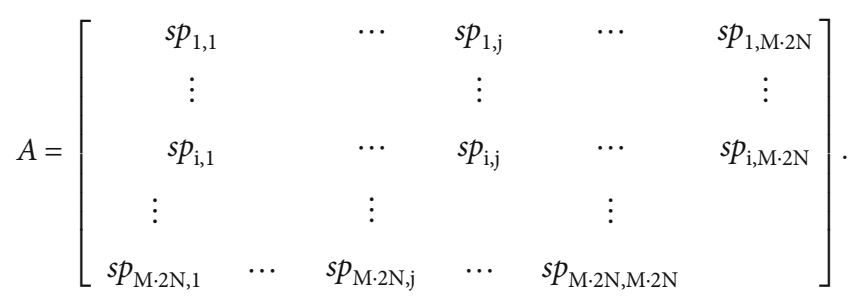

The matrix $B$ is

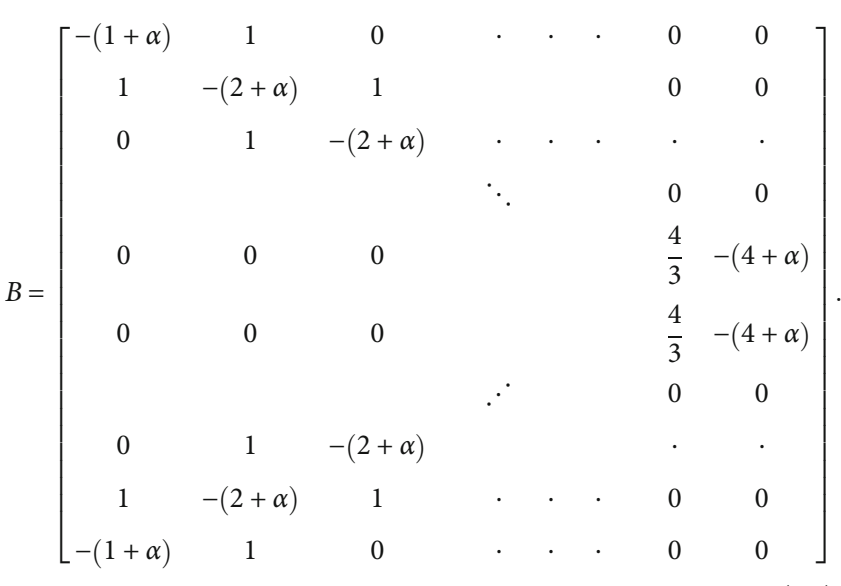

The matrix $C$ is $\beta \cdot I$, and $I$ is a unit matrix whose dimension is $M \cdot 2 N \times M \cdot 2 N$.

Solving Eq. (17) with Newton iteration method results in the bottom hole pressure and production distribution in Laplace domain space for any fracture long the multistage 


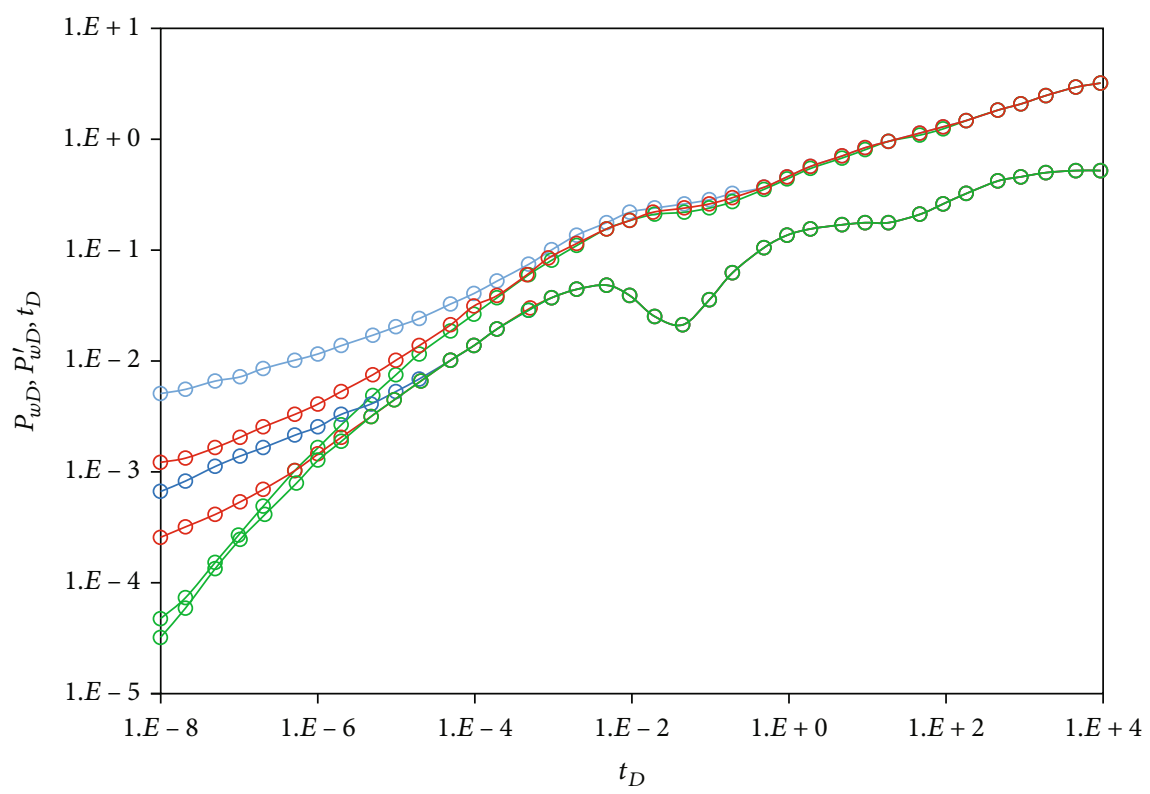

FIGURE 5: Effect of conductivity of fractures on pressure behavior of multistage fractured horizontal wells.

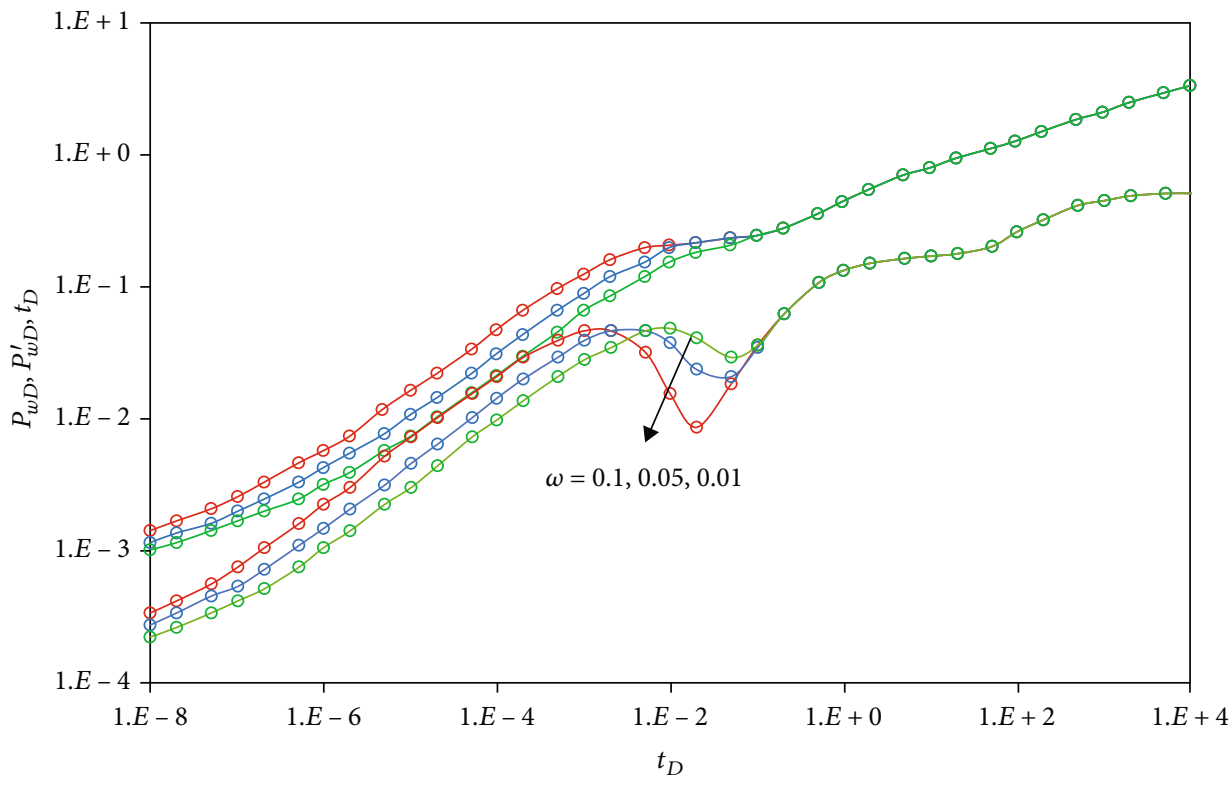

FIGURE 6: Effect of storability ratio on pressure behavior of multistage fractured horizontal wells.

fractured horizontal well. The solution in the real space is obtained by Stehfest inversion [24]. If the artificial fracture is viewed as infinitely conductive, Eq. (7) and Eq. (3) can be combined to solve the model for multistage fractured horizontal wells with infinite conductivity.

\section{Characteristic of Type Curves and Sensitivity Analysis}

The coupling equation is solved, and the type curves are constructed for the pressure and differential of pressure in multistage fractured horizontal wells in tight oil reservoirs as shown in Figure 4. There are six flow regimes: (1) the flow from the fracture into the wellbore, (2) the linear flow in the fracture (the first linear flow period), (3) the crossflow from the matrix to the fracture, (4) the radial flow around the fracture (the first radial flow period), (5) the linear flow from outside of the reservoir to fractured region (the second linear flow period), and (6) the boundary-dominant radial flow (the second radial flow period).

Figure 5 shows the effect of finite conductivity on the pressure behavior of multistage fractured horizontal wells in tight oil reservoirs. It is observed that the conductivity has a significant influence on the pressure behavior in the initial production period. The pressure and differential of pressure become smaller as the conductivity of fractures 


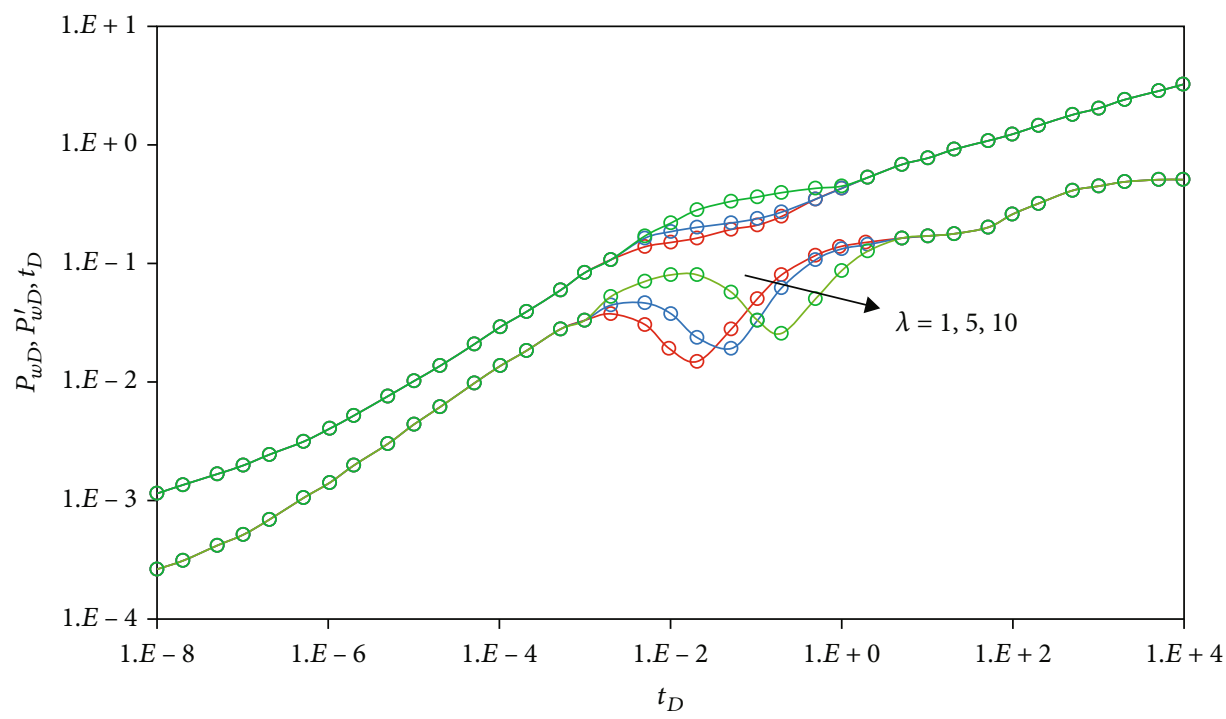

FIGURE 7: Effect of interporosity flow coefficient on pressure behavior of multistage fractured horizontal wells.

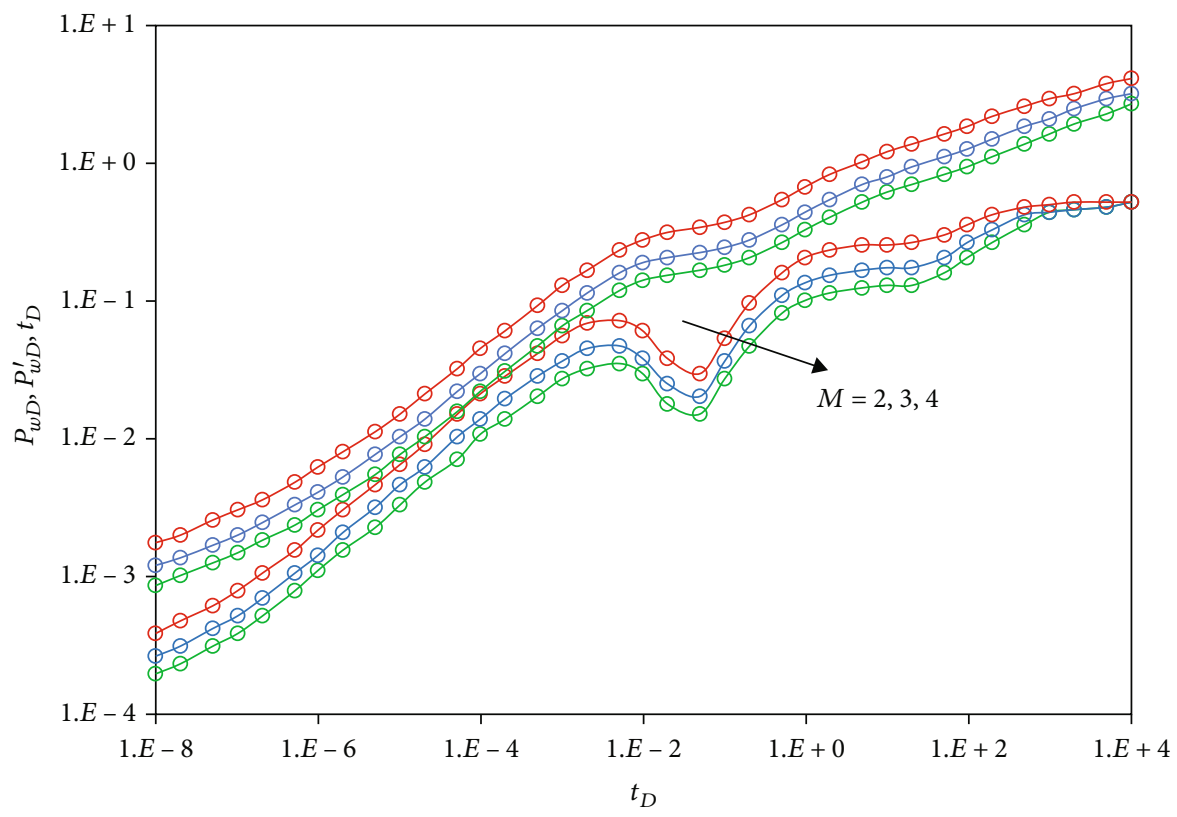

FIGURE 8: Effect of quantity of fractures on pressure behavior of multistage fractured horizontal wells.

increases. Once the conductivity is large enough, the pressure drawdown in the fracture can be neglected, and the model is reduced to infinite conductivity model. Therefore, increasing fracturing intensity is an effective way to maximize the initial productivity of fractured horizontal wells.

Figure 6 shows the effect of storability ratio on pressure behavior of multistage fractured horizontal wells in tight oil reservoirs. Storability ratio influences the duration of crossflow from the matrix to the fracture system. The duration increases, and the "concavity" gets deeper when storability ratio decreases.

Figure 7 shows the effect of the interporosity flow coefficient on pressure behavior of multistage fractured horizontal wells in tight oil reservoirs. The interporosity flow coefficient influences the duration of first linear flow, the time when the crossflow from the matrix to the fracture begins and the time when the first radial flow begins. As the interporosity flow coefficient decreases, the duration of first linear flow decreases, the "concavity" comes out later, and the first radial flow begins later. When the interporosity flow coefficient is big enough, the first radial flow is overwhelmed, and the flow regime switches to the second linear flow directly.

Figure 8 shows the effect of fracture quantity on pressure behavior of multistage fractured horizontal wells in tight oil reservoirs. The quantity of fractures has a significant influence on the pressure behavior of fractured horizontal wells 


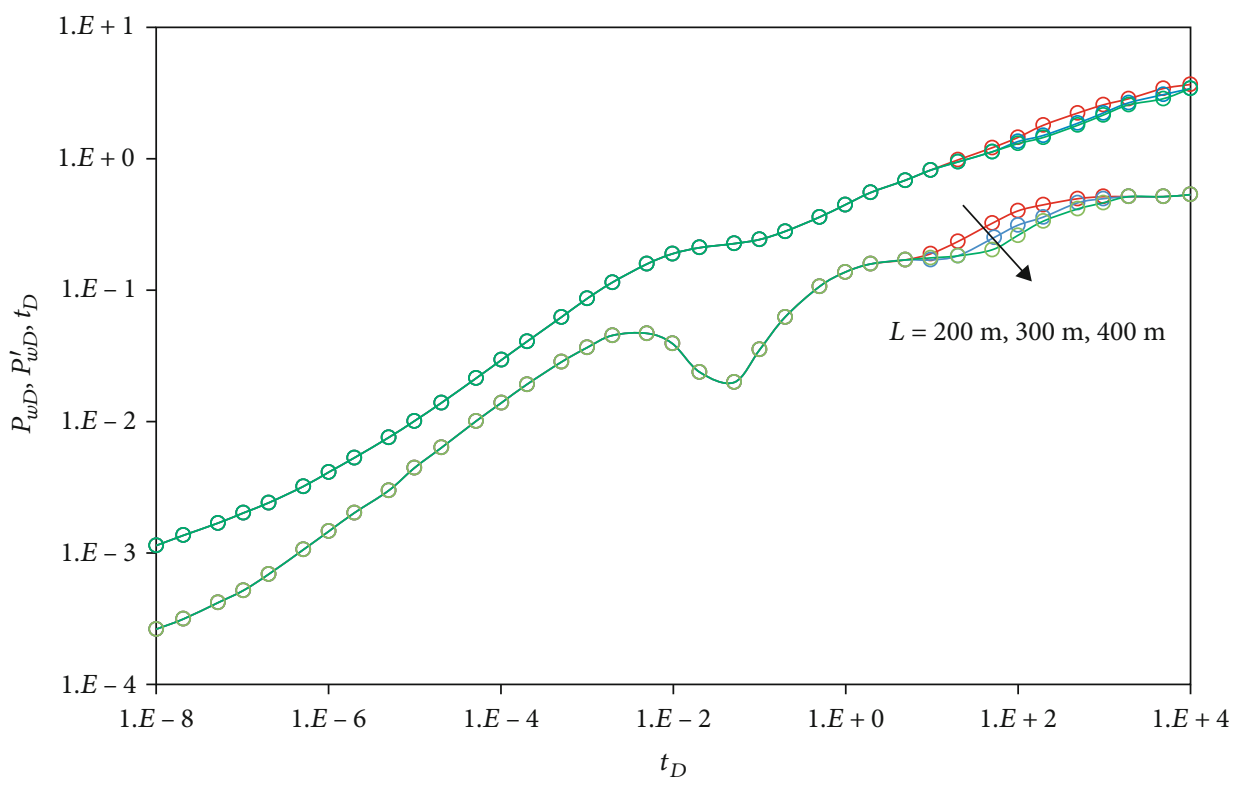

Figure 9: Effect of fracture spacing on pressure behavior of multistage fractured horizontal wells.

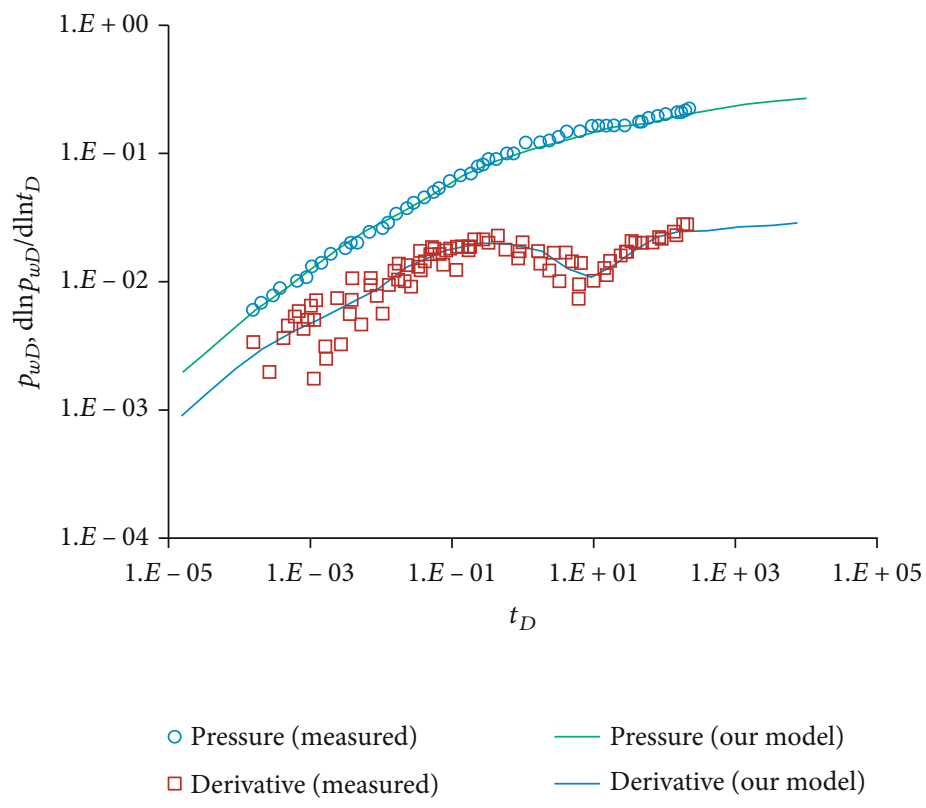

FIGURE 10: Fitting diagram of theoretical curve and actual curve of test well.

in the early and midterm period. The stimulated region near wellbore becomes larger under more fractures, and thus, the filtrational resistance decreases, and the productivity of fractured horizontal wells increases.

Figure 9 shows the effect of fracture spacing on pressure behavior of multistage fractured horizontal wells in tight oil reservoirs. The fracture spacing has a significant influence on the duration of first radial flow in the midterm to late period. The duration of the first radial flow increases as the fracture spacing increases. Once the fracture spacing is too small, the interference between fractures is intensified, and thus, the first radial flow period is overwhelmed.

\section{Field Example}

In order to verify the correctness of the model, a test case of an oil field is taken for verification. The basic parameters of the reservoir are as follows. The average effective thickness and porosity is $35 \mathrm{~m}$ and 0.18 . The wellbore radius is $0.1 \mathrm{~m}$. The horizontal interval length is $80 \mathrm{~m}$. Crude oil viscosity and volume coefficient are $2 \mathrm{MPa} \cdot \mathrm{s}$ and 1.02 . The comprehensive compressibility coefficient is $9.2 \times 10^{-4} \mathrm{MPa}^{-1}$, and pretest production is $54 \mathrm{~m}^{3} / \mathrm{d}$. The model presented in this paper is applied, and the genetic algorithm is used to automatically fit the theoretical pressure response and the 
measured pressure response. The fitting results are shown in Figure 10. The average reservoir permeability is $180 \times 10^{-4}$ $\mu \mathrm{m}^{2}$. The elastic storage capacity ratio is 0.199 . The channel flow coefficient is $9.89 \times 10^{-6}$. The fracture number is 3 , and the fracture half-length is $62 \mathrm{~m}$.

\section{Summary and Conclusions}

(1) A new analytical model for multistage fractured horizontal wells in tight oil reservoirs with dual-porosity medium is built, in which the fracture assumed to be finitely conductive. The type curves are constructed for pressure behavior in multistage fractured horizontal wells by numerical inversion

(2) The conductivity of artificial fractures has a significant influence on the initial production period of multistage fractured horizontal wells. As the conductivity increases, the pressure drawdown in fracture decreases, which leads to a higher initial productivity

(3) The storability ratio affects the duration of the crossflow from the matrix into the fracture. The duration of crossflow increases with the decrease of storability ratio. The interporosity flow coefficient influences the time when the fluid starts to flow from the matrix into the fracture. As the interporosity flow coefficient becomes smaller, the duration of the first linear flow decreases, the "concavity" comes out earlier, and the first radial flow period begins earlier

(4) The quantity of fractures has an influence on the midterm pressure behavior. The productivity of horizontal wells increases with the increase of fractures. The interference between fractures is intensified under smaller fracture spacing. When the fracture spacing is too small, the first radial flow period is overwhelmed

\section{Nomenclature}

$\phi: \quad$ Porosity, dimensionless

$p_{i}: \quad$ Initial formation pressure, $\mathrm{MPa}$

$x_{f}: \quad$ Half-length of artificial fracture, $\mathrm{m}$

$k_{f}: \quad$ Permeability of fracture, $\mathrm{mD}$

$w$ : $\quad$ Fracture width, $\mathrm{m}$

$q_{i, j}: \quad$ Flux per unit length of discrete segment $(i, j)$, $\mathrm{m}^{3} / \mathrm{s}$

$x_{m i, j}, y_{m i, j}:$ Coordinates of midpoint of discrete segment $(i, j)$

$L: \quad$ Spacing of hydraulic fractures, $\mathrm{m}$

$C_{f D}: \quad$ Dimensionless conductivity of hydraulic fractures

$\omega: \quad$ Storativity ratio

$\mu: \quad \quad$ Fluid viscosity, $\mathrm{mPa} \cdot \mathrm{s}$

$p_{W}: \quad$ Wellbore pressure, $\mathrm{MPa}$

$k: \quad$ Reservoir permeability, $\mathrm{mD}$

$h: \quad$ Reservoir thickness, $\mathrm{m}$

$t_{D}: \quad$ Dimensionless time

$(i, j): \quad$ The $j$ th discrete segment of the $i$ th fracture $x_{i, j}, y_{i, j}: \quad$ Coordinates of endpoint of discrete segment $(i, j)$

$M$ : $\quad$ Number of hydraulic fractures

$N: \quad$ Number of segments on the wing of each fracture

$\lambda: \quad$ Interporosity flow coefficient.

\section{Data Availability}

The (A Practical Solution Model for Transient Pressure Behavior of Multi-stage Fractured Horizontal Wells with Finite Conductivity in Tight Oil Reservoirs) data used to support the findings of this study are included within the article.

\section{Conflicts of Interest}

The authors declare that they have no conflicts of interest.

\section{Acknowledgments}

We acknowledge that this study was partially supported by the National Natural Science Foundation of China (No. 52004307), the Beijing Municipal Natural Science Foundation (No. 3204053), and the Science Foundation of China University of Petroleum, Beijing (No. 2462018YJRC015). We would further like to thank financial support of the National Natural Science Foundation of China (No. 51774297 and No. U1762210).

\section{References}

[1] H. Liu, Y. Luo, X. Li et al., "Advanced completion and fracturing techniques in tight oil reservoirs in Ordos Basin: a workflow to maximize well potential," SPE, 2012.

[2] M. J. Mayerhofer, E. P. Lolon, N. R. Warpinski, C. L. Cipolla, D. Walser, and C. M. Rightmire, "What is stimulated reservoir volume?," SPE Production \& Operations, vol. 25, no. 1, article 119890, pp. 89-98, 2010.

[3] J. E. Brown and M. J. Economides, "An analysis of hydraulically fractured. horizontal wells,” SPE, 1992.

[4] L. Larsen and T. M. Hegre, "Pressure transient analysis of multifractured horizontal wells," SPE Annual Technical Conference and Exhibition, 1994.

[5] T. M. Hegre and L. Larsen, "Productivity of multifractured horizontal wells," SPE, 1994.

[6] H. Li, Z. Jia, and Z. Wei, "A new method to predict performance of fractured horizontal wells," SPE, 1996.

[7] C.-C. Chen and R. Raghavan, "A multiply-fractured horizontal well in a rectangular drainage region," SPE Journal, vol. 2, no. 4, pp. 455-465, 1997.

[8] M. Al-Kobaisi, E. Ozkan, H. Kazemi, and B. Ramirez, "Pressure-transient-analysis of horizontal wells with transverse, finite-conductivity fractures," PETSOC, 2006.

[9] Z. Wang, H. Jin, and J. Wei, "Interpretation of the coupling model between fracture variable mass flow and reservoir flow for fractured horizontal wells," Chinese Journal of Hydrodynamics, vol. 24, no. 2, pp. 172-179, 2009.

[10] P. Lian, L. Cheng, R. Cao, and H. Shijun, “A couplong model for low permeability reservoir and fractured horizontal wellbore in nonsteady state," Chinese Journal of Computational Physics, vol. 27, no. 2, 2010. 
[11] H. Sun, J. Yao, P. Lian, D. Fan, and S. Zhixue, "A transient reservoir/wellbore coupling model for fractured horizontal wells with consideration of fluid from base rocks into wellbores," Acta Petrolei Sinica, vol. 33, no. 1, 2012.

[12] P. P. Valkó and S. Amini, “The method of distributed volumetric sources for calculating the transient and pseudosteady-state productivity of complex well-fracture configurations," SPE, 2007.

[13] D. Zhu, F. Magalhaes, and P. P. Valkó, "Predicting productivity of multiple-fractured horizontal gas wells," SPE, 2007.

[14] J. Lin and D. Zhu, "Predicting well performance in complex fracture systems by slab source method," SPE, 2012.

[15] S. Yao, F. Zeng, H. Liu, and G. Zhao, "A semi-analytical model for multi-stage fractured horizontal wells," Journal of Hydrology, vol. 507, pp. 201-212, 2013.

[16] Y.-l. Zhao, L.-h. Zhang, and W. Feng, "Pressure transient analysis for multi-fractured horizontal well in shale gas reservoirs," Journal of Petroleum Science and Engineering, vol. 90-91, pp. 31-38, 2012.

[17] J. Guo, L. Zhang, H. Wang, and G. Feng, "Retracted article: pressure transient analysis for multi-stage fractured horizontal Wells in shale gas reservoirs," Transport in Porous Media, vol. 93, no. 3, pp. 635-653, 2012.

[18] M. Brown, E. Ozkan, R. Raghavan, and H. Kazemi, "Practical solutions for pressure transient responses of fractured horizontal wells in unconventional reservoirs," SPE, 2009.

[19] I. Brohi, M. Pooladi-Darvish, and R. Aguilera, "Modeling fractured horizontal wells as dual porosity composite reservoirsapplication to tight gas, shale gas and tight oil cases," SPE, 2011.

[20] E. Stalgorova and L. Mattar, "Practical analytical method to simulate production of horizontal wells with branch fractures," SPE, 2012.

[21] E. Stalgorova and L. Mattar, "Analytical model for unconventional multifractured composite systems," Reservoir Evaluation \& Engineering, vol. 8, pp. 246-256, 2013.

[22] E. Ozkan and R. Raghavan, "New solutions for well-testanalysis problems: part 1-analytical considerations," SPE Formation Evaluation, vol. 6, no. 3, article 18615, pp. 359-368, 1991.

[23] L. Heber Cinco, V. F. Samaniego, and A. N. Dominguez, "Transient pressure behavior for a well with a finiteconductivity vertical fracture," Society of Petroleum Engineers Journal, vol. 18, no. 4, article 6014, pp. 253-264, 1978.

[24] H. Stehfest, "Algorithm 368: numerical inversion of Laplace transforms [D5]," Communications of the ACM, vol. 13, no. 1, pp. 47-49, 1970. 